Körpers zu erkennen. Die Malariaparasiten, die Parasiten des Texasfiebers, die Parasiten der Tsetsekrankheit, sie alle können heute in schöner und für den Histologen wie Kliniker gleich interessanter Weise leicht durch eine bestimmte Färbung zur Anschauung gebracht werden.

Diese Färbung, die Romanowski zuerst angewandt hat und die Ziemann und N,ocht dann genauer ausgearbeitet haben, stellt in genauester Weise den Bau dieser einzelligen thierischen Organismen dar und darf daher - ich komme darauf weiter unten noch zurück - als eine besonders geeignete Färbung für die einzelligen Organismen bezeichnet werden.

Diese jetzt allgemein bekannten Thatsachen sollen hier nur angeführt werden, um klarzulegen, dass man mit Hilfe der Technik und speziell der Färbung die wichtigsten und bedeutendsten Resultate bezüglich der Aetiologie der Krankheiten zu Tage gefördert hat. Es ist daher um so befremdender und um so erstaunenswerther, dass die geringsten Versuche, durch die Technik und speziell die der Färbung das Dunkel, das über die Ursache des schwierigsten Kapitels in der Pathologie, über die bösartigen Geschwülste herrschte, aufzuklären, géradezu eine Entrüstung verschiedener Pathologen hervorrufen. Statt durch genauen und kritischen Vergleich einzelne bemerkenswerthe $\mathrm{Be}$ funde, die bei dem Studium der bösartigen Geschwülste gewonnen werden, zu prüfen, erscheinen zumeist sofort nach einer Publikation derselben Mittheilungen, in denen diese Befunde anscheinend auf das eingehendste widerlegt werden.

Wenn man solche Arbeiten, die von Seiten einzelner Pathologen gegen bemerkenswerthe und mit der Aetiologie der Tumoren wohl zusammenhängende Befunde veröffentlicht werden, liest, so muss man sich unwillkürlich fragen, ist denn bisher noch nie ein Bacillus, noch nie ein Coccus, noch nie ein Parasit der Blutkrankheiten durch die Technik der Färbung dargestellt worden?

Seit meiner Veröffentlichung in No. 11 dieser Wochenschrift vorigen Jahrganges, in welcher ich dargelegt habe, dass in frischen Krebsgeschwülsten, in denen noch keine Degenerationsprodukte sich zeigten, einzellige selbständige Organismen vorkommen, die von allen Kernen und Kernfiguren enthaltenden Einschlüssen der "Krebszellen" scharf zu differenziren sind und die sich dann auch in den zerfallenen Geschwülsten, die ich untersuchte, fanden, haben meine Befunde die verschiedensten Auslegungen erhalten. Als erster hat Herr Geh. Rath O. Hertwig sich dahin ausgesprochen (No. 13 dieser Wochenschrift 1902), dass die von mir angegebenen Momente, welche bestimmte Zellen als einzellige Or-

V. Aus dem botanischen Institut der landwirthschaftlichen Hochschule und dem pflanzenphysiologischen Institut der Universität Berlin.

\section{Ueber das Gewebe und die Ursache der Krebsgeschwülste.}

\section{Von Dr. L. Feinberg.}

Seitdem die Technik unserer mikroskopischen Untersuchungsmethoden, speziell die der Färbungen eine so ausgezeichnete geworden, wie wir sie nun schon seit einer Reihe von Jahren kennen, ist denjenigen mikroskopischen Forschungen, welche die ursächlichen Erscheinungen der verschiedenen Krankheitsprozesse im menschlichen Körper zu ergründen suchten, eine ergiebige und fruchtbare Quelle geliefert worden.

Das ganze Gebäude der Bakteriologie, dessen neuester und für die Menschheit wichtigster Zweig, die Serumgewinnung, sich nunmehr auf chemischem Gebiete bewegt, ist, wenn ich so sagen darf, aufgebaut auf dieser Technik; denn durch dieselbe mussten zuerst der Tuberkelbacillus, der Diphtheriebacillus, der Pneumococcus, der Gonococcus und andere im Gewebe in ihrer Struktur erkannt und als Bacillus oder Coccus festgestellt werden, bevor man dazu überging, diese Bakterien zu züchten und mit Hilfe dieser Culturen am Thiere zu experimentiren.

Aber auch ein grosses Gebiet anderer Krankheitsprozesse, die nicht bakterieller Natur waren, konnte erst und bisher sogar allein durch die Technik der Färbung in ihrer Aetiologie erforscht und erschlossen werden, die Blutkrankheiten. - Nicht allein, dass wir mit Hilfe der Ehrlich'schen Färbemethoden perniziöse von leichten Anämieen zu unterscheiden im Stande sind; alle einzelligen, selbständigen thierischen Organismen, die im Blute des Menschen und Thieres pathologisch vorkommen, vermögen wir heute durch eine bestimmte Färbemethode zu unterscheiden und in ihrem ganzen Entwickelungscyklus innerhalb des menschlichen ganismen charakterisirten, hierzu "nicht im entferntesten genügten“, Hertwig glaubt vielmehr, dass es sich um "Vakuolen“ handeln würde, die "durch die Fixation und durch die Dreifärbung der Schnitte künstlich hervorgerufen würden".

Sodann ist 0. Lubarsch in seiner Broschüre: "Pathologische Anatomie und Krebsforschung" gegen verschiedene Autoren, unter anderen auch gegen mich zu Felde gezogen. Nach seiner Ansicht ist der von mir beschriebene einzellige Organismus ein in Karyorrhexis befindlicher Kern. „Jedenfalls", so meint Lubars ch, „ist die Aehnlichkeit mit dem, was Schmaus und Albrecht ${ }^{1}$ ) in ihrer Arbeit über Karyorrhexis abbilden, eine frappante“.

Vor kurzem ist schliesslich durch eine besonders bemerkenswerthe Veröffentlichung, die, aus der Feder des hervorragenden Pathologen, Herrn Geh. Rath Marchand stammend, in den letzten Nummern dieser Wochenschrift") erschienen ist,. mit - ich darf wohl sagen - wenigen Sätzen über diejenigen bisherigen Arbeiten, die sich mit der Aetiologie der Krebsgeschwülste beschäftigt haben, der Stab gebrochen worden. Marchand hält diese im Krebsgewebe auffallenden Gebilde im Gegensatz zu Lubarsch und Hertwig für "durch eine Art innerer Sekretion entstandene Vakuolen".

Es liegt mir fern, die Ansichten von Ribbert, von v. Hansemann, welche diese Autoren der Entstehung der malignen Tumoren zu Grunde legen, die sich jedoch von einander wieder unterscheiden, hier zu erörtern, fern, die so geistvollen theoretischen Betrachtungen Marchand's über die Genese der Geschwülste bei den geringen Erfahrungen, die ich gegenüber den wohl Jahrzehnte umfassenden Forschungen unseres bekannten Pathologen besitze, irgend welcher Kritik zu unterziehen. Nur das, was als thatsächlich bestehend zu allen diesen

1) Virchow's Archiv Bd. CXXXVIII, Suppl.

2) Deutsche medizinische Wochenschrift 1902, No. 39 u. 40. 
Theorieen, die doch immer und immer nur Theorieen bleiben, in schärfstem Contrast steht, möchte ich an dieser Stelle kurz zusammenfassen.

Den exakten Forschungen derjenigen, die sich mit dem Studium der Histologie und Aetiologie der bösartigen Geschwülste beschäftigt haben, hat es zum grossen Nachtheil gereicht, dass eine grosse Anzahl von Autoren, die nach ihren Arbeiten entweder eine oberflächliche oder gar keine Kenntniss von Protozoën, Myxomyceten oder Blastomyceten zu haben scheinen, Mittheilungen über die verschiedenartigsten Befunde von Krebsparasiten machten. Es musste thatsächlich ein grosses Misstrauen erwecken, wenn in jedem Jahre ein anderer Organismus als der Erreger der Krebsgeschwülste ohne die geringste Kritik und Prüfung hingestellt wurde. Es haben daher auch natürlich die wenigen Arbeiten, die durch ihre Forschung eine genaueste Nachprüfung ihrer Befunde von den verschiedensten Seiten nothwendig erscheinen liessen, von vornherein so wenig Zutrauen erweckt, dass man sie vielfach gleichfalls mit wenigen Worten widerlegte. Man warf auf diese Weise sämmtliche Arbeiten, die über die Histologie und Aetiologie der Krebsgeschwülste geschrieben sind, wenn ich mich so ausdrücken darf, in einen Topf; bei ihrer Kritik haben freilich verschiedene Autoren, v. Hansemann, Lubarsch ausdrücklich stets versichert, dass sie „nicht Gegner der parasitären Aetiologie der Geschwülste" wären. Gebot eine solche Bemerkung noch eine gewisse Vorsicht, so hat Marchand in seiner letzten Veröffentlichung sich rückhaltlos als Gegner der parasitälen Entstehung der Tumoren bekannt und gemeint, „dass es wohl zeitgemäss wäre, das aussichtslose Suchen nach Carcinomparasiten aufzugeben." Auch noch durch einen zweiten Punkt unterscheidet sich die Arbeit Marchand's von denjenigen Mittheilungen, welche bisher die Entstehung der $\mathrm{Ge}$ schwülste durch einen Parasiten für höchst unwahrscheinlich darstellen. Marchand hat nämlich aus allen den vielen Publikationen, die über den Krebs und andere Geschwülste geschrieben sind, in im allgemeinen richtiger Erkenntniss alle diejenigen ausgeschaltet, die deutlich den "Stempel eines groben Irrthums" tragen, und nur diejenigen Arbeiten über die Aetiologie der Geschwülste einer kurzen Besprechung unterzogen, welche in exakter Weise, wenn auch in gewissen Intervallen, ähnliche Befunde gebracht haben. Hierbei hat Marchand betont, dass thatsächlich „gewisse Körperchen in den Krebsgeschwülsten vorkommen, die in grösserer Zahl einen frappanten Eindruck machen."

Nach meiner Ansicht waren es nur vier Autoren, die gewisse Gebilde in den Krebsgeschwiilsten beschrieben haben, die von thatsächlicher Bedeutung für die Aetiologie der Tumoren werden dürften: Soudakewicz, ${ }^{1}$ Foa, ${ }^{2}$ ) Plimmer ${ }^{3}$ ) und Sawtschenko ${ }^{4}$ ).

Es ist ohne Frage, dass diese vier Autoren Organismen innerhalb des Krebsgewebes beschrieben und auch durch Abbildung dargestellt haben, die von allen im Krebsgewebe vorkommenden und, wenn ich so sagen darf, zu demselben gehörenden Formen sich so unterscheiden, dass sie als fremde Organismen imponirten.

Es mag hier dahingestellt bleiben, ob alle die Beschreibungen und Darstellungen, die diese Autoren in ihren Arbeiten gebracht haben, Formen des Entwickelungscyklus eines einzelligen Organismus darstellen; es soll hier auch nicht erörtert werden, ob sie alle mit einander identifizirt werden können und ob sie, was aus den Abbildungen von Soudakewicz und Sawtschenko z. B. ohne weiteres hervorgeht, nicht auch Zellen als Parasiten darstellen, die als zufällige Befunde gedeutet werden müssen.

Nur das muss hervorgehoben werden, dass allen diesen Mittheilungen ein thatsächlicher Beweis dafür fehlt, dass die verschiedenartig gestalteten Gebilde, die diese Autoren in ihren Abbildungen zur Anschauung gebracht haben, wirklich einzellige thierische (pflanzliche?) Organismen waren.

Dies geht schon allein aus dem Umstande hervor, dass Plimmer z. B. dieselben Gebilde in einer Arbeit als Protozoën, in einer anderen als Sprosspilze (!) deutet, ein sicheres Kriterium des Baues dieser Organismen also gänzlich fehlt!

Die verschiedenen Angriffe, die sich auch gegen diese Arbeiten erhoben, konnten daher nicht widerlegt werden.

1) Annales de l'Inst. Pasteur 1892. Tome VI.

2) Archives italiennes, Tome XX, Fasc. 1. Turin 1893.

3) Société de Biologie 1893, p. 836.

4) Bibliotheca medica 1895, Abtheilung D, Heft 9.
Als ich mich im Jahre 1896 in Folge eines zufälligen $\mathrm{Be}^{-}$ fundes ${ }^{1}$ ) auf der I. medizinischen Universitätsklinik ${ }^{2}$ ) dem Studium der malignen Tumoren zuwandte, war es meine erste Aufgabe, mich eingehend mit der Struktur und dem Bau der einzelligen selbständigen Organismen zu beschäftigen. Dieses Studium hat nun zu dem Ergebniss geführt, dass der Kern der Hauptklassen der einzelligen thierischen (pflanzlichen?) Organismen eine Ausnahmestellung unter allen Zellen der Thier- und Pflanzenwelt einnimmt. Ich verweise hierbei auf folgende meiner Arbeiten, die sämmtlich diesen Gegenstand zum Kardinalpunkt ihres Inhaltes haben.

„Ueber Amöben und ihre Unterscheidung von Körperzellen.“ (Fortschritte der Medizin 1899, Heft III.) „Ueber den Erreger der krankhaften Auswüchse des Kohls" (Deutsche medizinische Wochenschrift 1902, No. 3).

"Ueber die Unterscheidung des Kerns der Pflanzenzellen von dem Kern der einzelligen thierischen Organismen" (Berichte der deutschen botanischen Gesellschaft 1902, Heft V).

„Ueber den Bau der Ganglienzelle und über die Unterscheidung ihres Kerns von dem Kern der einzelligen thierischen Organismen" (Monatsschrift für Psychiatrie und Neurologie 1902, Heft 6).

„Ueber den Bau der einzelligen thierischen Organismen und über ihre Unterscheidung von den Körper- und Pflanzenzellen" (Berliner klinische Wochenschrift 1902, Heft 24).

In diesen verschiedenen Publikationen glaube ich den Beweis dafür erbracht zu haben, dass sich für den Bau der untersuchten einzelligen thierischen (pflanzlichen?) Organismen in Bezug auf ihren Kern im grossen und ganzen ein allgemeines Gesetz auifstellen lässt. Denn während wir wissen, dass der Kern der Zellen des Thier- und Pflanzenkörpers aus einer Kernmembran, einem Kerngerüst und einem oder mehreren Kernkörperchen besteht, finden wir bei den einzelligen thierischen (pflanzlichen?) Organismen niemals Nukleolen und nichts von einem Kerngerüst. In den von mir bisher untersuchten einzelligen thierischen Organismen habe ich überhaupt nichts von einer Nukleolarsubstanz in dem Kern der Protozoën gefunden.

Hingegen ist die Substanz, die das Kerngerüst der thierischen (pflanzlichen?) Körperzellen ausmacht, die Chromatinsubstanz, in analoger Weise bei den einzelligen thierischen Organismen vorhanden, jedoch in völlig anderer Form. Die untersuchten einzelligen thierischen (pflanzlichen?) Organismen haben nämlich im allgemeinen einen mehr oder minder kleinen compakten Chromatinkörper, der im ruhenden Zustande mehr oder minder rund, ist. Ich habe diesen Chromatinkörper als den „Kernpunkt“ bezeichnet.

Zweitens finden wir im Contrast zu dem Kern der Körperund Pflanzenzellen, dass dieser „Kernpunkt“ (Chromatinsubstanz) bei den Protozoën von einer ungefärbten Zone umgeben ist, die, im Ruhezustande des betreffenden Thieres betrachtet, völlig inhaltlos erscheint. Es ist daher wohl ausser Frage, dass diese den „Kernpunkt“ (compakte Chromatinsubstanz) umgebende Zone, die den eigentlichen Kern von dem Protoplasma trennt, aus dem Kernsaft gebildet wird.

Diese für die grosse Mehrzahl der thierischen (pflanzlichen?) Organismen so charakteristische Kernform konnte ich sowohl in frischem Material als in verschiedenen Färbungen bei einer grossen Anzahl von Protozoën nachweisen (conf. meine Arbeiten) Besonders beweiskräftig hierfür dürfte die Anwendung der $\mathrm{Me}$ thylenblau-Eosin- (Romanowski'schen) Färbemethode sein.

Durch diese Anwendung der Romanowski'schen Färbemethode gelang es mir nämlich, eine scharfe differentielle Färbung des Nucleolus und des Kerngerüstes (Chromatinsubstanz) der Körper- und Pflanzenzellcn zu ermöglichen. Denn während sich die Chromatinsubstanz nach dieser Färbung (MethylenblauEosin) intensiv roth-violett färbt, giebt das Kernkörperchen (Nucleolus) bei dieser Färbung eine intensiv blaue Tinktion, und zwar auffallender Weise ebenso wie das Protoplasma der Zellen. Diese Thatsache lässt siclı bei allen Zellen, die

1) Leydenia gemmipara Schaudinn. Akademie der Wissenschaften 1890, Heft 39.

3) Conf. meine Arbeit: Zur Lehre des Gewebes und der Ursache der Krebsgeschwülste. Deutsche medizinische Wochenschrift 1902, No. 11.

3) Centralblatt für Bakteriologie, Abth, I, 1899 
einen Nucleolus besitzen, feststellen. Besonders charakteristisch konnten mit dieser Färbemethode auch die Ganglienzellen zur Darstellung gebracht werden, deren Kerngerüst (Chromatinsubstanz) bei sonstigen Färbungsmethoden bekanntlich sehr schwer darstellbar ist. Da auch die Untersuchung der Pflanzenzellen (Vitia faba) eine genaue Uebereinstimmung dieses Unterschiedes des Kerngerüstes und des Kernkörperchens lieferte, so haben wir in dieser Methylenblau-Eoisin-Färbemethode eine sehr schöne Unterscheidung der Nukleolarsubstanz von der Chromatinsubstanz. Die Anwendung dieser Färbemethode bei verschiedenen Protozoën ergab nun, dass bei den untersuchten einzelligen thierischen Organismen auch nicht eine Spur einer Nukleolarsubstanz vorhanden ist und dass der als Kern sichtbare runde, mehr oder minder compakte „Kernpunkt“ dieser einzelligen thierischen Organismen nur aus Chromatinsubstanz besteht und allseitig von dem inhaltlosen Kernsaft umgeben ist.

Es ist hier nicht der Ort, noch weiter auf den Bau der einzelligen thierischen und pflanzlichen Organismen einzugehen, zumal dieselben zur Zeit noch einem genaueren Studium unter der persönlichen Leitung des Herrn Geh. Rath Eilhard Schulze im hiesigen zoologischen Institut von mir unterworfen werden.

Von der Thatsache dieser in dem ganzen Gebiete der thierischen und pflanzlichen Zellen einzig dastehenden Kernform der einzelligen selbständigen Organismen ausgehend, hätte ich nun ein Moment, das als ein Charakteristikum dieselben von allen Zellen des Thier- und Pflanzenkörpers auf das schärfste und auf das prägnanteste unterscheidet. Es lag daher bei der histologischen Betrachtung der Krebsgeschwülste wohl der Gedanke nahe, auf dieses thatsächlich bestehende Unterscheidungsmoment mein Augenmerk zu richten. Wir wissen nun, dass in den Krebsgeschwülsten eine Anzahl Degenerationsformen vorkommen (die colloiden, die hyalinen Degenerationsformen), die schon vielfach $\mathrm{zu}$, ich darf wohl sagen, groben Irrthümern Veranlassung gegeben haben. Wir wissen ferner, dass degenerirte Kerne in den malignen Tumoren sich vielfach finden, die gleichfalls als merkwürdige Bilder sehr imponirten und die selbst von ausgezeichneten Forschern irrthümlicher Weise als irgend ein Stadium eines einzelligen Thieres angesehen wurden.

Durch die Technik der Fixation, Einbettung wie Färbung ist es mir nun gelungen, in Präparaten von Krebsgeschwülsten, die völlig frei von irgend welchen Degenerationsformen waren, Gebilde $\mathrm{zu}$ erhalten, die ausser dem Zellkörper (bestehend aus einer scharf contourirten Membran und einem radiär gestreiften, den ziemlich grossen Inhalt der Zelle ausfüllenden Protoplasma diejenige Form und Bau des Kerns besitzen, welcher den einzelligen thierischen Organismen nach meinen Untersuchungen zukommt.

Solche Zellen, die ohne Frage den Ruhezustand eines einzelligen thierischen Organismus darstellen, habe ich weder in der gesammten Litteratur, noch bei einem der vorher genannten vier Autoren durch eine Beschreibung der Abbildung dargestellt gefunden!

Ich habe es absichtlich vermieden, bei den so vielfach widersprechenden Ansichten und Meinungen vieler Pathologen und Histologen auch andere Zellen in der Krebsgeschwulst, die höchstwahrscheinlich dem Entwickelungscyklus dieses thierischen Organismus angehören und die wohl in Analogie zu bringen sind mit einem Theil derjenigen Beschreibungen, die die vier vorher genannten Autoren mitgetheilt haben, zunächst als thierischen Organismus $\mathrm{zu}$ bezeichnen oder zu beschreiben. Ich habe nur die Zelle als sicheren thierischen (pflanzlichen?) Organismus hingestellt, die durch den Bau und die Form ihres Kerns mich hierzu berechtigte. Um jedoch dem Einwand von vornherein $\mathrm{zu}$ begegnen, dass die Form dieser Gebilde durch irgend welche Degenerationen, die in den Krebsgeschwülsten bei längerem Bestand vielfach auftreten, zu Stande kommen könnten, habe ich Tumorstückchen, die möglichst kurze Zeit (natürlich, soweit es klinisch festgestellt werden konnte) bestanden hatten, ganz frisch bei der Operation fixirt, um dieselben mikroskopischen Untersuchungen zu unterwerfen. Ich habe in ihnen dann keine Degenerationsprodukte wahrnehmen können. Wenn 0. Lubarsch $^{1}$ ) daher behauptet, dass schon aus der Beschreibung meiner histologischen Präparate eine Auflösung in der Krebsstruktur sich bemerkbar macht, so widerspricht diese Bemerkung absolut

\footnotetext{
1) Pathologische Anatomie und Krebsforschung. Wiesbaden 1902.
}

nicht meiner Angabe, dass in der histologischen Untersuchung derselben keine Degenerationsprodukte zu finden waren. Diese histologische Diagnose, die Lubarsch aus der Beschreibung neiner Präparate macht, dürfte übrigens neu sein. Auch an anderer Stelle überrascht Lubarsch Zoologen wie Botaniker in gleicher Weise. Lubarsch hält es nämlich für ganz sicher, dass "Protozoën und Myxomyceten keineswegs identisch seien". Oder sollte die Meinungsverschiedenheit, die unter Botanikern und Zoologen in diesem Punkte herrscht und die von einem so ausgezeichneten Forscher wie Doflein vor kurzem dahin entschieden ist, dass die Myxomyceten den Protozoën zuzutheilen sind, Lubarsch nicht bekannt sein? Die Art der Einwendungen, die Lubarsch überhaupt gegen mich zu machen sucht, ist in der That eine eigenthümliche $\mathrm{zu}$ nennen! Ich habe nämlich in meiner ersten Veröffentlichung ausdrücklich hervorgehoben, dass alle Kulturversuche von keinem sicheren Erfolg eher gekrönt sein werden. als bis es gelungen ist, die angeblichen Parasiten in den Geschwülsten selbst aufs sicherste nachzuweisen. Denn, geben einerseits die verschiedenen und vielfachen Mittheilungen wie meine eigenen Erfahrungen bei den Kulturversuchen der Krebsgeschwülste ein untrügliches Zeichen dafür, dass es möglich ist, die verschiedensten Protozoën, Bakterien und Sprosspilze aus den malignen Tumoren zu züchten, so ist es doch andererseits selbstverständlich, dass Jemand, der in Carcinomen vorhandene Parasiten züchtet, dieselben doch zuerst im Gewebe nachweist. Diese meine Ansicht hält Lubarsch zum mindesten für „bedenklich."!

Dann möchte ich noch kurz auf die Bemerkung von Lubarsch eingehen, dass die von mir in Krebsgeschwülsten beschriebenen einzelligen Organismen in Karyorrhexis begriffene Kerne sind. Ja, Lubarsch behauptet sogar, dass die von Schmausl) in seiner Arbeit gebrachten Abbildungen mit meinen Parasiten eine „frappante Aehnlichkeit" hätten. Ich überlasse Jedem, der den Vergleich der von mir beschriebenen Organismen mit den von Schmaus gebrachten Abbildungen macht, das Urtheil darüber, wie weit diese Behauptungen von Lubarsch richtig sind oder nicht. In keinem Stadium zeigt ein in Karyorrhexis befindlicher Kern der Körperzelle auch nur annähernd den von mir beschriebenen Bau und die Form des Kerns der einzelligen thierischen Organismen. Was schliesslich noch die Bemerkung von Lubarsch betrifft, dass man ,getrost sagen kann, dass sich bei einem "Theil der Krebsgeschwülste“, die ich untersucht habe, Degenerationsprodukte fanden", so habe ich dies nie in Abrede gestellt. Im Gegentheil habe ich die Darmcarcinome ausdrücklich zur Untersuchung mit herangezogen, um mich zu überzeugen, dass sich auch in Tumoren, welche Degenerationsprodukte zeigten, diese von mir beschriebenen einzelligen thierischen Organismen, wenn auch in geringerer Anzahl fanden

Im Gegensatz zu Lubarsch haben nun Herr Geheimrath o. Hertwig und Herr Geheimrath Marchand behauptet, dass die von mir beschriebenen einzelligen Organismen Vakuolen sind. Diese Vakuolen sollen nach der Ansicht von O. Hertwig durch die „Fixation und die Dreifärbung der Schnitte“ künstlich, nach der Ansicht von Marchand dagegen "durch eine Art innerer Sekretion" entstanden sein, die eine "wechselnde Menge gerinnungsfähiger Substanz" enthalten!

Es ist von gewissem Interesse, dass die Ansichten zweier so hervorragenden Forscher bei der Auslegung dieser Gebilde contrastiren und dass beide keine thatsächliche Erklärung derselben zu geben im Stande sind. Denn, glaube ich dem Einwand des Herrn Geheimrath Hertwig, dass die von mir beschriebenen, im Krebsgewebe vorkommenden einzelligen thierischen Organismen durch die Kunst hervorgerufene Vakuolen sind, dadurch völlig zu begegnen, dass dieselben durch andere Fixationsmethoden (Alkohol absolutus) und durch andere Färbungen (Methylenblau, Methylenblau-Eosin) dargestellt werden konıten, so entbehrt der Einwand Marchand's einer Begründung. Denn Marchand macht zweierlei gegen mich geltend, einmal dass der von mir aufgestellte Satz über den Bau des Kerns der einzelligen thierischen Organismen nicht richtig sei, "weil der Kern der einzelligen Protozoën die grössten Verschiedenheiten aufweise“. Diese Bemerkung Marchand's entspricht in keiner Weise den Thatsachen. In dem Ruhezustande zeigt der Kern der einzelligen thierischen organismen (bei der überwiegenden 
Mehrzahl) gesetzmässig den von mir oben beschriebenen Bau und Form.

Ich werde Herrn Geheimrath Marchand in einer weiteren Arbeit diese Thatsache noch auf das eingehendste nachweisen! Als zweiten Punkt macht Herr Geheimrath Marchand, folgendes gegen mich geltend: „Für den Fall, dass diese Zelle ein thierischer Organismus wäre, würde man doch, so meint Herr Geheimrath Marchand, vergeblich nach dem zugehörigen Zellkörper suchen". Ich darf Herrn Geheimrath Marchand darauf hinweisen, dass ich in meiner Arbeit das Plasma auf das genaueste beschrieben habe. Ich bin auch gern bereit, Herrn Geheimrath Marchand Präparate vorzulegen, in denen das Protoplasma dieser einzelligen Organismen in anschaulicher und sich von dem eigenthümlichen Kern unterscheidender Form aufs deutlichste $\mathrm{zu}$ erkennen ist.

Durch meine letzten Mittheilungen in der Berliner klinischen Wochenschrift ${ }^{1}$ ), in denen ich die Thatsache publizirte, dass es mir gelungen war, die einzige Färbemethode, durch die wir heute die einzelligen Parasiten des Blutes auf das prägnanteste nicht nur erkennen, sondern selbst in ihrem Entwickelungsstadium genau studiren können, die Methylenblau-Eosin-Färbung, auch bei den Gewebsschnitten im allgemeinen und speziell bei den Krebsgeschwülsten anzuwenden, und dass auch bei dieser Färbemethode Resultate erzielt wurden, die völlig meinen ersten Mittheilungen über die in den Krebsgeschwülsten vorkommenden einzelligen Organismen entsprachen, glaube ich nun einen einwandsfreien Beweis dafür erbracht zu haben, dass gewisse in den Krebsgeschwülsten vorkommende einzellige Organismen selbständige sind. Die genaue Einreihung derselben in das grosse Gebiet der einzelligen thierischen (pflanzlichen?) Organismen und die Feststellung ihres Entwickelungsganges werden wohl weitere Forschungen noch ergeben.

Nur das kann hier bereits hervorgehoben werden, dass die Identifizirung dieser in den Krebsgeschwülsten vorkommenden einzelligen Organismen mit verschiedenen Parasiten des Thier- und Pflanzenkörpers, wie es immer wieder in oberflächlichster Weise geschieht, z. B. mit dem Erreger der Kohlhernie (!), auf die Kenntnisse und Forschungen solcher Autoren ein merkwürdiges Licht wirft. Durch derartige Mittheilungen werden die Arbeiten über die Histologie und Aetiologie der Geschwülste immer wieder gehemmt. Denn das einzige thatsächlich zu Vergleichen der verschiedensten einzelligen Parasiten mit den in den Krebsgeschwülsten vorkommenden selbständigen Organismen Anlass gebende Moment ist nach meinen bisherigen Untersuchungen allen diesen einzelligen thierischen (pflanzlichen?) Organismen gemeinsam, der Bau und die Form ihres Kerns! 